\title{
Down-regulation of mechanisms involved in cell transport and maintenance of mucosal integrity in pigs infected with Lawsonia intracellularis
}

\author{
Sionagh H Smith ${ }^{1}$, Alison D Wilson ${ }^{2}$, Imke Van Ettinger ${ }^{1}$, Neil Maclntyre ${ }^{1}$, Alan L Archibald ${ }^{2}$ and Tahar Ait-Ali $^{2^{*}}$
}

\begin{abstract}
Lawsonia intracellularis is an obligate intracellular bacterium, responsible for the disease complex known as proliferative enteropathy (PE). L. intracellularis is associated with intestinal crypt epithelial cell proliferation but the mechanisms responsible are yet to be defined. Microarray analysis was used to investigate the host-pathogen interaction in experimentally infected pigs to identify pathways that may be involved. lleal samples originating from twenty-eight weaner pigs experimentally challenged with a pure culture of L. intracellularis (strain LR189/5/83) were subjected to microarray analysis. Microarray transcriptional signatures were validated using immunohistochemistry and quantitative real time PCR of selected genes at various time points post challenge. At peak of infection (14 days post challenge) $86 \%$ of altered transcripts were down regulated, particularly those involved in maintenance of mucosal integrity and regulation of cell transport. Among the up-regulated transcripts, CD163 and CDK1 were novel findings and considered to be important, due to their respective roles in innate immunity and cellular proliferation. Overall, targeted cellular mechanisms included those that are important in epithelial restitution, migration and protection; maintenance of stable inter-epithelial cell relationships; cell transport of nutrients and electrolytes; innate immunity; and cell cycle.
\end{abstract}

\section{Introduction}

Lawsonia intracellularis is an obligate intracellular, Gram negative bacterial pathogen that causes the disease complex known as proliferative enteropathy (PE). In terms of economic impact this disease is primarily a problem for the pig industry but it also occurs in several other species, though much more sporadically [1-4]. In weaner and grower pigs, clinical signs consist of non-haemorrhagic diarrhoea, ill-thrift and reduced growth rates but in older pigs the consequences of infection can include fulminant intestinal haemorrhage and death $[5,6]$. In severe cases, $\mathrm{PE}$ is characterised by macroscopically obvious thickening of the intestinal mucosal lining which is due to proliferation of epithelial cells which line intestinal crypts, mainly in the distal ileum, though not restricted to it $[7,8]$. There is a consistent close association between this proliferation and the intracytoplasmic presence of $L$. intracellularis in the hyperplastic crypt epithelial cells [9].

\footnotetext{
* Correspondence: tahar.aitali@roslin.ed.ac.uk

${ }^{2}$ The Roslin Institute, University of Edinburgh, Easter Bush Campus, Roslin, Midlothian EH25 9RG, UK

Full list of author information is available at the end of the article
}

Koch's postulates were proven in the 1990s and subsequent research has pursued diverse paths, ranging from the development of molecular-based diagnostic tests to the evaluation of various control and treatment options [10-14]. Despite this progress, important mechanistic questions remain unanswered, including the cause of the pathognomonic proliferative lesion and why there are such distinct clinical manifestations of the disease in two different age-groups. There has been limited exploration of the host/pathogen interaction at the cellular level, probably due to the fact that $L$. intracellularis has such rigorous growth requirements $[15,16]$. The relationship between $L$. intracellularis and the associated crypt proliferation seems to be unique and its potential value as a model for studying the cell cycle, and even the molecular pathways that ultimately lead to cancer, has been recognised for some time $[17,18]$. Initial analyses of host transcriptional responses to infection using microarrays or RNA-seq support the theory that cell cycle proteins and growth factors are altered in cells infected with $L$. intracellularis $[16,19,20]$. A number of bacterial 
pathogens have been associated with cellular proliferation and have been discussed in this context previously $[16,21]$. One of the main goals of the study reported here was to identify changes in gene expression that might shed some light on host-pathogen interactions occurring in PE. The particular strengths of this work were the analysis of transcript alterations in pigs at several time points following experimental infection with a known strain of cultured L. intracellularis, using a comprehensive microarray platform capable of monitoring 47000 porcine transcripts.

\section{Materials and methods Intestinal tissues}

Samples of frozen ileum originated from a previous challenge study [22]. Briefly, 28 seven-week-old pigs were randomly selected from a minimal-disease herd, penned in seven groups of four pigs each and tested for various enteric pathogens. Faecal samples from all animals were culture negative for Brachyspira hyodysenteriae, B. pilosicoli, Yersinia spp. and Salmonella spp., and PCR negative for $L$. intracellularis $[22,23]$. All pigs were also serologically negative for $L$. intracellularis. The pigs were challenged orally with a pure culture of $L$. intracellularis (isolate LR189/5/83), euthanased and subjected to a full necropsy at $3,7,14,21,28,35$ or 42 days post challenge (dpc), with four pigs per time point. A full-thickness sample of ileum was collected from each pig, snap frozen at $-95^{\circ} \mathrm{C}$ using isopentane and dry ice, fixed to a cork disk with optimal cutting temperature compound and stored at $-80^{\circ} \mathrm{C}$. The results have been described fully by MacIntyre et al. [22]. For the current study a separate group of three uninfected age-matched pigs was used as controls to provide base line data for the gene expression analyses.

\section{Extraction of total genomic DNA from ileum}

Total genomic DNA was extracted from $~ 100 \mathrm{mg}$ of ileum from 24 pigs using a standard salt extraction method incorporating proteinase K. The quantity of each DNA sample was assessed using a Nanodrop spectrophotometer (NanoDrop Technologies Inc, Wilmington, DE, USA) and the quality was assessed using agarose gel electrophoresis.

\section{Quantification of $L$. intracellularis-specific genomic DNA}

In order to allow measurement of $L$. intracellularis-specific genomic DNA in the total genomic DNA samples, a standard curve for quantitative real time PCR (qPCR) was constructed using a pGEM $^{\circledR}$-T Vector plasmid which contained a $322 \mathrm{bp}$ L. intracellularis ribosomal $16 \mathrm{~S}$ rRNA gene insert [24]. Serial dilutions of the amplified plasmid were analysed by qPCR using the following primers as previously described [25]: Forward primer, 5 '-GCGCGCG TAGGTGGTTA-3'; reverse primer, 5' -GCCACCCTCTC CGATACTCA-3' and platinum SYBR Green PCR SuperMix
UDG (Invitrogen, Paisley, UK). The thermocycling profile used on a Stratagene Mx3000 was as follows: $50{ }^{\circ} \mathrm{C}$ for $2 \mathrm{~min}, 95^{\circ} \mathrm{C}$ for $2 \mathrm{~min}, 40$ cycles of $95{ }^{\circ} \mathrm{C}$ for $15 \mathrm{~s}$ and $60{ }^{\circ} \mathrm{C}$ for $30 \mathrm{~s}$. The profile of the final cycle was $95{ }^{\circ} \mathrm{C}$ for $1 \mathrm{~min}, 60{ }^{\circ} \mathrm{C}$ for $30 \mathrm{~s}$ and $95{ }^{\circ} \mathrm{C}$ for $15 \mathrm{~s}$. The standard curve was used to estimate the $L$. intracellularis-specific genomic DNA concentration using DNA of sufficient quantity and quality from the ileal samples (24 of the 28 pigs) and the concentration was expressed as number of $16 \mathrm{~S}$ rRNA copies per ng of DNA.

\section{RNA extraction}

Total RNA was extracted from the ileum of 20 pigs using Trizol (Invitrogen, Paisley, UK) according to standard methods [26]. The RNA was cleaned using the Qiagen RNeasy minikit following the manufacturers' instructions (Qiagen, Crawley, UK). RNA was eluted from the spin column in $30 \mu \mathrm{L}$ of RNase-free water and stored as aliquots at $-80{ }^{\circ} \mathrm{C}$. The quantity and quality of RNA were assessed using a Nanodrop spectrophotometer (NanoDrop Technologies Inc, Wilmington, DE, USA) and Agilent 2100 bioanalyser (Agilent Technologies, Palo Alto, CA, USA).

\section{Quantitative real time PCR validation}

The differential expression of several selected genes, as identified from the microarray data, was verified at various time points using qPCR. Reverse transcription was performed as described previously [26,27]. Briefly, one microgram of total RNA was reverse transcribed using a TaqMan kit (Applied Biosystems, Foster City, CA, USA). For qPCR, Platinum SYBR Green PCR SuperMix UDG was used, as described above. The qPCR was performed with a Stratagene MX3000P (Stratagene, La Jolla, CA, USA). Samples were tested in triplicate, GAPDH served as the housekeeping gene and results were calculated as described previously [26,27]. Primers used are listed in Additional file 1.

\section{Microarray platform and data analysis}

To assess if host transcriptional responses were affected, the L. intracellularis-infected ileal tissues described above $(3,7,14,21,28$ and $42 \mathrm{dpc})$ were analysed using the Affymetrix Snowball GeneChip ${ }^{\circledR}$ [28]. Sense-strand cDNA was generated from total RNA (500 ng) and subjected to two rounds of amplification (Ambion ${ }^{\circledR}$ WT Expression Kit). The resulting cDNA was used for biotin labelling and fragmentation according to the Affymetrix GeneChip ${ }^{\circledR}$ WT Terminal Labelling and Hybridization protocol (Affymetrix UK, High Wycombe). Biotin-labelled fragments of cDNA $(5.5 \mu \mathrm{g})$ were hybridized to Affymetrix SNOWBALL arrays using the Affymetrix HybWashStain kit and manufacturer's recommendations. After hybridization, the arrays were washed and stained using the Affymetrix 
Fluidics Station 450 and then scanned in an Affymetrix $7 \mathrm{G}$ scanner. Image generation and the resulting CEL files for analysis were produced in AGCC - Affymetrix GeneChip Command Console Software. Initial QCs were performed in Expression Console. All microarray data used in the analyses herein are freely available from the Array Express repository under the accession number E-MTAB1396 [29]. The Affymetrix.CEL files were imported into the Genomics Suite software package version 6.13.0213 (Partek software, Partek Inc.) for data analysis. Transcriptional responses were normalised to those from agematched uninfected pig ileum prior to running an ANOVA analysis of the data. Up-regulated and down-regulated differentially expressed transcripts at each dpc were selected for further consideration if the false discovery rate (FDR) was $\leq 0.1$.

\section{Gene ontology and pathway analysis}

Gene ontology and pathway analysis were carried out using DAVID bioinformatics resources and Ingenuity pathway analysis, respectively [30,31]. Gene expression data were obtained using BioGPS [32].

\section{CD163 immunohistochemistry}

Sections of formalin fixed ileum from all four pigs euthanased at $14 \mathrm{dpc}$, three pigs euthanased at $42 \mathrm{dpc}$ and one uninfected control pig were stained immunohistochemically to detect CD163 antigen expression. Briefly, after antigen retrieval with proteinase K (Dako UK Ltd., Ely, UK) for $10 \mathrm{~min}$ at room temperature, endogenous peroxidase activity was blocked using a commercial blocking agent for $10 \mathrm{~min}\left(\mathrm{REAL}^{\mathrm{mm}}\right.$ peroxidase blocking agent, Dako UK Ltd., Ely, UK S202386). Following serial washes, sections were incubated with mouse-anti-pig CD163 monoclonal antibody (Serotec Ab MCA2311) diluted 1:30 in Tris buffered saline Tween at pH 7.5. They were incubated with labelled polymer for $40 \mathrm{~min}$ at room temperature (Envision mouse HRP reagent, Dako K4001), treated with 3, 3'-diaminobenzidine and counterstained with haematoxylin. Unrelated porcine ileum containing a known macrophage population as defined by a previous study served as a positive control [22]. For each section, the density of immunopositive cells was calculated using image analysis (Olympus Soft Imaging System, Münster, Germany). For each of six randomly selected high power fields $(400 \times)$ a field of interest was outlined, consisting only of lamina propria, and its area calculated. The number of CD163-positive cells within that specified area was counted per high power field. An aggregate density was calculated for each pig by dividing the total number of CD163 positive cells by the total area examined. A Kruskal-Wallis test was used to compare the aggregate densities for the pigs in the $14 \mathrm{dpc}$ group with those in the $42 \mathrm{dpc}$ group. Assessment of these particular time points allowed comparison of the density of CD163 positive cells at peak infection with the density when infection had virtually resolved. The CD163-positive cell density at $14 \mathrm{dpc}$ was also tested for evidence of a direct correlation with infection burden, as measured by qPCR of L. intracellularis genomic DNA. This time point was selected as it corresponded with the highest fold alteration in CD163 gene expression in the microarray analysis.

\section{Results}

\section{Quantification of $L$. intracellularis bacterial load}

L. intracellularis bacterial load was measured in the ileum of infected pigs at 3, 7, 14, 21, 28, 35 and $42 \mathrm{dpc}$ using a qPCR assay of the 16S rRNA gene. The sensitivity of the qPCR test approximated to $\log _{10}(0.06)$ copies per ng of DNA. Figure 1 illustrates the pattern of $16 \mathrm{~S}$ rRNA gene accumulation, with a peak in mean burden at $14 \mathrm{dpc}$, falling to undetectable levels at $28 \mathrm{dpc}$. With the exception of $7 \mathrm{dpc}$ the difference between the infection burden at $14 \mathrm{dpc}$ and other time points were statistically significant.

\section{Host transcript regulation during $L$. intracellularis infection}

The integrity numbers for the RNA samples used in the microarray were above 6 . In the microarray analysis, the number of transcripts which changed significantly $(p<0.1)$ from uninfected levels was the greatest at $14 \mathrm{dpc}$ (Table 1 and Additional file 2). At all the time points, the number of transcripts that were down-regulated was greater than those that were up-regulated, with peak fold changes broadly occurring at $14 \mathrm{dpc}$.

\section{Validation of differentially regulated transcripts using quantitative real time PCR}

Seven differentially regulated transcripts at $14 \mathrm{dpc}$ (fold change $\geq 2.0$, FDR $\leq 0.1$ ) were validated by qPCR analysis of RNA samples collected at 3, 7, 14, 21, 28 and $42 \mathrm{dpc}$. The selected genes were SLC7A9, S100G, SLC6A4, SLC13A1, $M U C 2$, TGFBR1 and CD163. The coefficient of correlation $\left(R^{2}\right)$ for each of these time points was produced by plotting the relative level of gene expression as assessed by microarray against the transcript levels generated by qPCR (Table 2, Additional files 2 and 3). With the exception of data at $7 \mathrm{dpc}$, the microarray and qPCR results appeared to be positively correlated (Table 2).

Gene ontology (GO) and Ingenuity pathway analysis (IPA) Transcripts differentially regulated in L. intracellularisinfected enterocytes were analysed with respect to their biological and molecular functions based on the gene ontology data available on DAVID bioinformatics resources (Table 3). Only down-regulated transcripts at $14 \mathrm{dpc}$ showed significant fold functional enrichment as follows: 


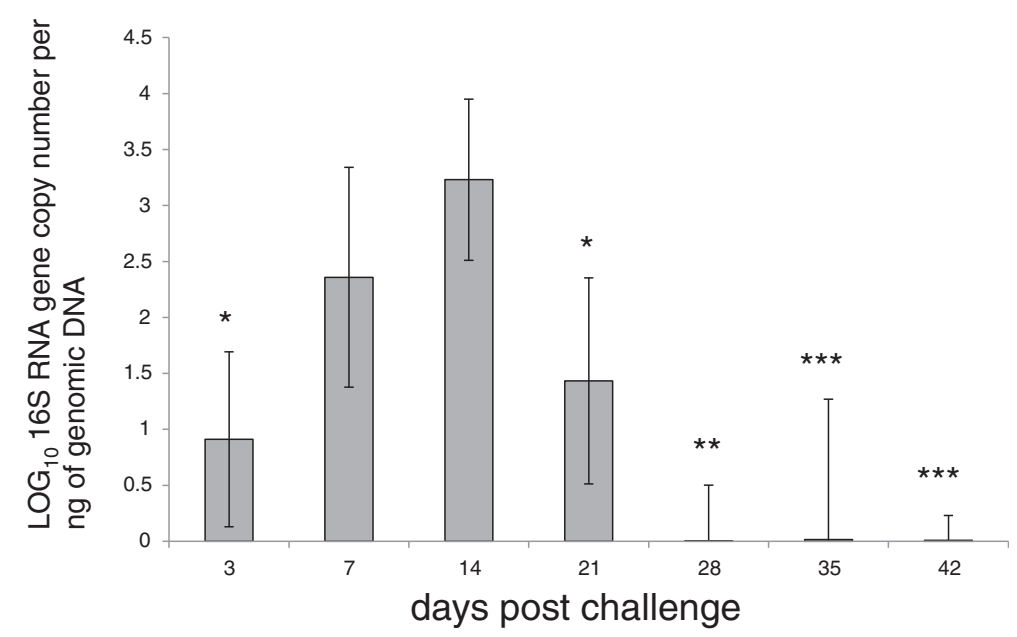

Figure 1 Quantification of 16S rRNA Lawsonia intracellularis LR187/5/83 in experimentally challenged pigs. 16S rRNA gene copy number per ng of genomic DNA was measured by qPCR as described in Materials and methods. The mean copy number of the 165 rRNA gene was expressed in Log10 for each time point assessed at $3(n=3), 7(n=4), 14(n=4), 21(n=4), 28(n=4), 35(n=2)$ and $42(n=3)$ days post challenge. For age-matched negative controls, $16 \mathrm{~S}$ rRNA gene was not detected (data not shown). Mean \pm standard error are shown. Two-tailed student $t$-test is shown for the most significant comparisons with $14 \mathrm{dpc}$ : $, P<0.02{ }^{*}{ }^{*}, P<0.006$ and ${ }^{* * *}, P<0.0009$.

solute/cation symporter activity (9, FDR (false discovery rate) $<2 \mathrm{E}-02)$, digestion $(16, \mathrm{FDR}<8.01 \mathrm{E}-06)$, intestinal absorption (37, FDR $<4 \mathrm{E}-02)$ and brush border membrane (27, FDR < 6.3E-04) (Additional file 4). Our results suggest that four broad functional categories were influenced by the presence of $L$. intracellularis. These were cellular transport; brush border/mucosal integrity; local immunity/ repair; and cell cycle. Down-regulated genes involved in cell transport included several solute carrier family members (SLC7A9, SLC26A3, SLC15A1, SLC13A1 and SLC6A4), CUBN (encoding cubilin, a transporter of cobalamin) and S100G, a gene encoding a calcium transporter, which was down-regulated more than 22 -fold at time of peak infection (14 dpc) compared to uninfected pigs. Down-regulated genes involved in brush border and mucosal integrity included $C U B N$ (suggesting a dual role for this gene), $M U C 2$, RETNLB, two members

Table 1 Summary of transcript changes at various time points post challenge (pc)

\begin{tabular}{cccc}
\hline Time pc (days) & $\begin{array}{c}\text { Number of } \\
\text { up-regulated } \\
\text { genes }\end{array}$ & $\begin{array}{c}\text { Number of } \\
\text { down-regulated } \\
\text { genes }\end{array}$ & $\begin{array}{c}\text { Total number } \\
\text { of transcripts } \\
\text { changed }\end{array}$ \\
\hline 3 & 0 & 1 & 1 \\
7 & 1 & 3 & 4 \\
14 & 26 & 159 & 185 \\
21 & 6 & 16 & 22 \\
28 & 19 & 18 & 37 \\
42 & 2 & 3 & 5 \\
\hline
\end{tabular}

FDR $<0.1$. of the trefoil factor family (TFF2 and TFF3) and several members of the solute carrier family. Some gene expression alterations indicated that there was suppression of local immunity, specifically a 2- to 3-fold down-regulation of several T-cell receptor complex genes (CD3 delta, epsilon and gamma molecules). In contrast, the greatest fold change in the up-regulated genes was observed in CD163, which encodes a protein expressed by scavenger macrophages [33]. We also recorded a 3-fold up-regulation in SLA-3 (MHC class I antigen) and, finally, the up- and down-regulation of CDK1 and HEPACAM2 transcript levels, respectively, both of which influence cellular proliferation.

\section{CD163 regulation during $L$. intracellularis infection}

Since an increase in intestinal macrophages has been previously associated with $L$. intraellularis infection, an immunohistochemical detection method was used to test if the CD163 transcript up-regulation was associated with a concomitant increase in macrophages specifically expressing the CD163 scavenger receptor protein [22]. At $14 \mathrm{dpc}$, there were moderate to large numbers of CD163 positive cells diffusely scattered throughout the lamina propria of the ileum in all four pigs (Figure 2A). At $42 \mathrm{dpc}$ and in the one negative control pig ileum that was immunostained, only very small numbers of CD163 positive cells were present in the lamina propria (Figure 2B). Contrary to expectation, the difference in densities between the $14 \mathrm{dpc}$ pigs and the $42 \mathrm{dpc}$ pigs was not statistically significant $(p=0.48)$. However, there was a positive correlation between the density of CD163 positive cells 
Table 2 Comparison between microarray and quantitative real time PCR assays

\begin{tabular}{lcccccc}
\hline Days post infection & 3 & 7 & 14 & 21 & 28 & 42 \\
$\mathrm{R}^{2}$ & 0.85 & 0.48 & 0.91 & 0.83 & 0.94 & 0.87 \\
\hline
\end{tabular}

Seven regulated transcripts with a FDR $<0.05$ were randomly selected at 14 dpi. Each primer pair was run with each RNA sample at 3, 7, 14, 21, 28 and 42 dpi. The square of the sample correlation coefficient $\left(R^{2}\right)$ between the fold changes observed in the qPCR assays and microarray data sets is shown (Additional file 3). Beta-actin transcript levels were used for data normalisation and RNA levels in mock infected ileum tissues were used for normalisation (see in Materials and methods).

and infection burden in the $14 \mathrm{dpc}$ group (correlation = 0.79), measured as $L$. intracellularis $16 \mathrm{~S}$ rRNA copies per nanogram of total genomic DNA (Figure 3).

\section{Discussion}

Only three previous studies have focused on host gene expression in the context of PE $[16,19,20]$. The first of these studies mapped transcript changes over three time points using cultured mouse McCoy fibroblast-like cells as in vitro hosts [19]. This mesenchymal cell line is superior to others in its capacity to generate large numbers of $L$. intracellularis bacteria but extrapolation of its expression profile to the porcine crypt enterocyte (the natural target cell) may not be reliable $[15,34,35]$. The three time points were also assessed much earlier than the time of peak lesion development in experimentally infected pigs $[21,36]$. Nevertheless, that study provided valuable baseline data pertaining to early bacterial invasion and indicated that, even at this stage of infection, there was altered transcription of genes involved in cell cycle control.

The second study used intestinal tissues from field cases of pigs with diarrhoea to investigate cytokine expression, also using microarray methodology [20]. This provided whole animal information, something that cell culture models cannot, but the study was confounded by co-infection with porcine circovirus type 2 (PCV2) and, since diseased pigs were field cases, the findings were not correlated with stage of infection.
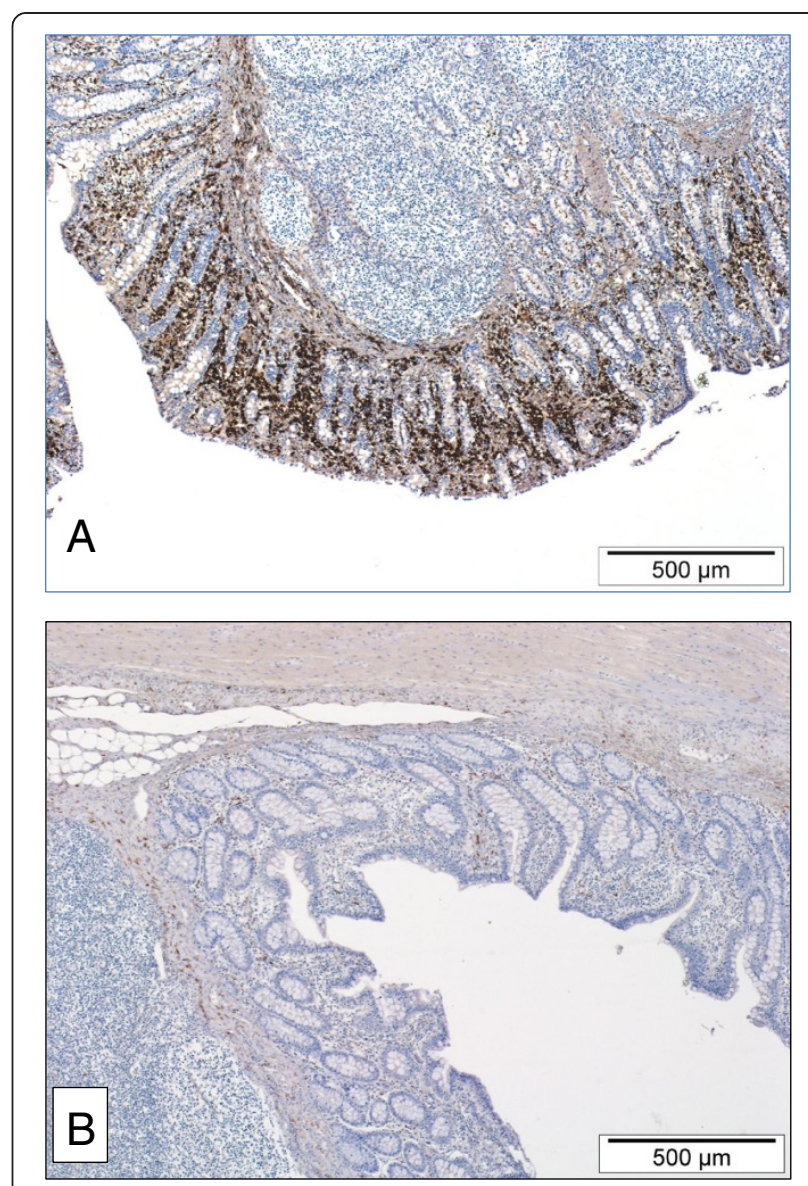

Figure 2 Pig ileum 14 days post challenge (A) and 42 days post challenge (B). Large numbers of CD163-expressing macrophages diffusely infiltrate the lamina propria of ileum collected from a pig at $14 \mathrm{dpc}(\mathbf{A})$. This is in contrast to the much smaller numbers of CD163-expressing macrophages in the lamina propria of ileum collected from a pig at $42 \mathrm{dpc}$ (B). Envision HRP and haematoxylin counterstain.

Table 3 Altered cellular networks at 14 dpc identified using ingenuity pathway analysis

\begin{tabular}{lll}
\hline Network functions & Score & Focus molecules \\
\hline Cancer, Gastrointestinal Disease, Cellular Function \& Maintenance & 53 & 25 \\
Lipid Metabolism, Molecular Transport, Small Molecule Biochemistry & 44 & 22 \\
Cellular Function \& Maintenance, Developmental Disorder, Endocrine System Disorders & 28 & 16 \\
Cell Morphology, Organ Morphology, Molecular Transport & 27 & 15 \\
Endocrine System Disorders, Reproductive System Disease, Developmental Disorder & 24 & 14 \\
Energy Production, Lipid Metabolism, Small Molecule Biochemistry & 20 & 12 \\
Gastrointestinal Disease, Inflammatory Disease, Inflammatory Response & 20 & 12 \\
Cell-To-Cell Signalling and Interaction, Cell Signalling, Behaviour & 10 & 7
\end{tabular}

Eight cellular networks most frequently associated with altered gene expression in L. intracellularis infected pig ileum. As described by [31], the network function score ranks the network and takes into account the number of Network Eligible molecules in the network and its size, as well as the total number of Network Eligible molecules analysed and the total number of molecules in the Ingenuity Knowledge Base that could potentially be included in networks. 


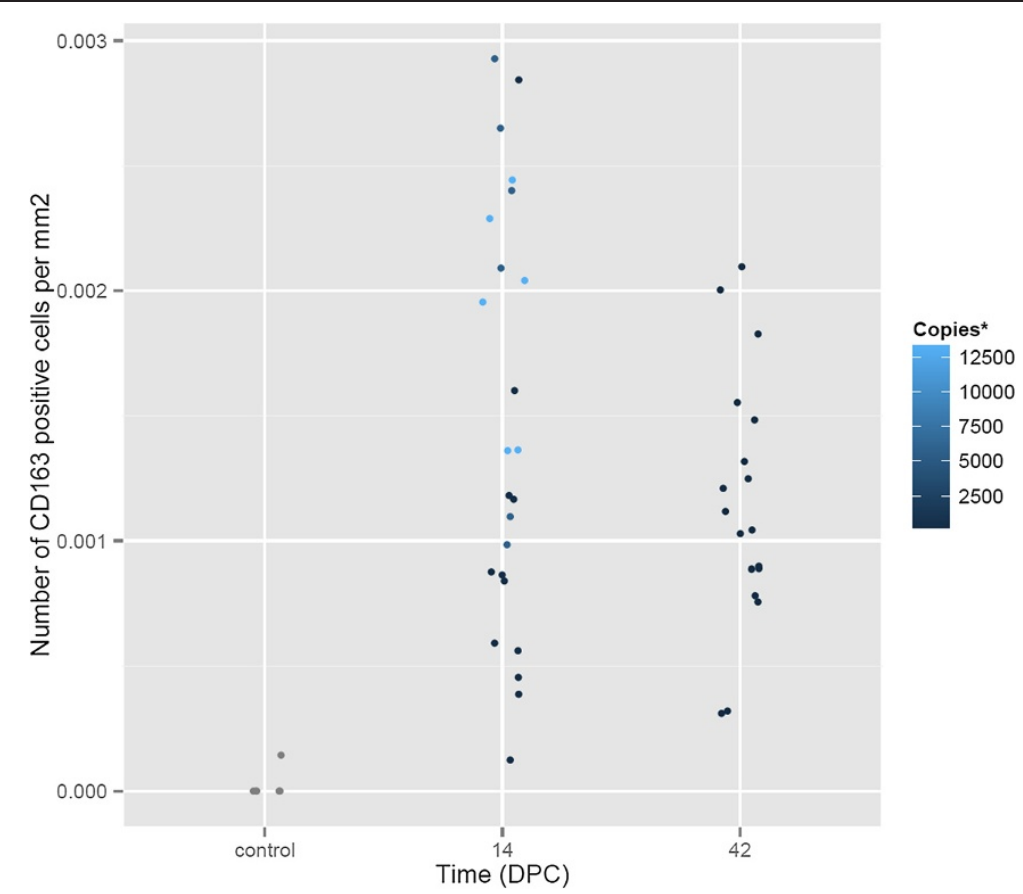

Figure 3 Density of CD163 positive macrophages in pigs 14 and 42 days post challenge. Comparison of the density of CD163 positive macrophages in the lamina propria of pig ileum infected with $L$. intracellularis 14 and 42 days post challenge (dpc). The colour gradations $(*)$ denote the infection burden, measured using $\mathrm{qPCR}$, as number of $L$. intracellularis $16 \mathrm{~S}$ rRNA copies per nanogram of total genomic DNA.

The most recent study introduced the use of laser capture microdissection in combination with RNA-seq analyses, allowing specific analysis of infected intestinal crypts from pigs experimentally challenged with a pure culture of L. intracellularis (strain PHE/MN1-00), negating any risk of confounding enteric infections. This novel approach facilitated the isolation of the cell of interest (crypt enterocyte) from the natural host, enabling a glimpse of the transcriptional alterations that may result from a combination of host and pathogen effects. Compared to that study, in which analysis was performed at a single time point coinciding with peak of infection, our own study examined multiple time points through initiation, peak and resolution of lesions. Specifically, we measured fold changes in gene expression for six weeks following challenge, allowing correlation with the previously described morphological lesions and level of infection in the same cohort of pigs [22]. In contrast to Vannucci et al. [16], we used a well-characterised comprehensive microarray platform which comprises over 47000 probesets [28]. We also extended the interrogation to surrounding host tissues, specifically the intestinal lamina propria, where immune responses in particular are likely to be manifest.

Quantitative real time PCR analysis of L. intracellularisspecific genomic DNA detected peak infection at $14 \mathrm{dpc}$, correlating with previous reports $[21,22,36]$. There were some differences in the estimates of the infection burden between our qPCR data and the immunohistochemistry data reported by MacIntyre et al. [22]. Our qPCR assays detected infection in $80 \%$ of pigs tested between 21 and $35 \mathrm{dpc}$, whilst MacIntyre et al. [22] confirmed infection in only $25 \%$ of pigs tested at similar time points, using IHC. Since we tested tissues from the same cohort of pigs as MacIntyre et al. [22], the difference in detection rate cannot be due to differences in bacterial strain or source of pig. Rather, it is more likely that qPCR is more sensitive than IHC for detection of infection in frozen tissue. There is also accepted variation between PCR and immunologically based tests that further depends on whether tissue, faeces or blood are tested [37,38]. Different depths within the tissue block were also used for DNA extraction and IHC, which may have contributed to variability.

We found statistically significant changes in transcript levels for a total of 218 separate genes. The assumption that any cellular changes triggered by L. intracellularis are maximally expressed at time of peak infection burden (14 $\mathrm{dpc}$ in this study) was substantiated by fold changes that reached their maximum at this time point in most measured transcripts. At $14 \mathrm{dpc}$ there were changes in 185 transcripts, $86 \%$ of which were down-regulated, a trend similar to that observed by Jacobson et al. [20]. This low number of altered transcripts is surprising, particularly as we used a highly comprehensive method that should have enhanced our ability to detect changes in gene expression 
[28]. One possible reason for this low detection level is individual host variation, intensified by the fact that the disease is unlikely to progress at exactly the same rate in different animals, as indicated in Figure 1, where there is variability in the accumulation of $L$. intracellularis $16 \mathrm{~S}$ rRNA. The only way to overcome this using the same model would be to increase the number of pigs in each group, which is not realistic with a large animal model such as the pig. A more speculative reason is that the pathogen has a low impact on the host due to coevolution or because it acts via a limited number of pathways that still have regulatory consequences for the infected cell. Further investigations of gene expression would help to determine whether this low level of transcriptional alteration and the predominance of down-regulated genes are genuine phenomena. Comparison of gene expression in intestinal tissues from pigs infected with a more aggressive pathogen, such as Salmonella or Clostridium spp., may also improve understanding in this regard.

We found the greatest fold down-regulation in S100G transcripts, the product of which is S100 calcium binding protein $\mathrm{G}$ (calbindin $\mathrm{d} 9 \mathrm{k}$ ). The precise role of S100G is uncertain but it facilitates intestinal absorption and transport of calcium under the influence of vitamin D [39]. None of the three previous analyses reported changes in this gene $[16,19,20]$. Down-regulation of various members of the solute carrier family (SLC) was also evident (Table 4). Solute carriers are membrane proteins responsible for the transport of nutrients and electrolytes. Those altered in our analysis are collectively responsible for the transport of oligopeptides, glucose, zinc, amino acids and neurotransmitters or the exchange of electrolytes, including $\mathrm{Na}^{+}$and $\mathrm{H}^{+}$[40]. The expression of $C U B N$, which encodes a vitamin B12 transporter, was also down-regulated. While a few of these changes have been previously reported, our analysis expanded the list of SLC family member genes that are differentially expressed in infected pigs [16]. Vannucci et al. [16] detected downregulation of $S L C 7 A 3$ and up-regulation of $S L C 2 A 1$, findings that we were not able to confirm as these particular genes are not present on the Snowball microarray [28]. The down-regulation of genes controlling transport of nutritional elements could be due to failure of maturation of mucosal epithelial cells and may contribute to malabsorption diarrhoea, as previously suggested [16]. Our study found concomitant down-regulation of SLC26A3, a chloride/bicarbonate exchanger, and SLC9A3, the gene encoding NHE3. NHE3 is a sodium/hydrogen ion exchanger that is expressed by gastrointestinal epithelial cells and that facilitates absorption of sodium by enterocytes. Since these exchangers work in parallel and are essential for the regulation of intestinal water absorption, we postulate that, as a consequence of infection with L. intracellularis, their down-regulation leads to reduced sodium absorption and osmotic diarrhoea [41]. In our study several genes involved in regulating intestinal mucosal integrity, notably MUC2 (encodes mucin-2), RETNLB (resistin-like beta), TFF2 and TFF3 (trefoil factors 2 and 3), and CLDN15 (claudin 15) were down-regulated. Mucin-2 (the principal secretory mucin), resistin-like beta and the trefoil peptides

Table 4 Comparison of solute carrier family gene expression changes between this study and Vannucci et al. [16]

\begin{tabular}{|c|c|c|c|c|}
\hline Gene symbol & This study & Vannuci et al. [16] & solute carrier function & $\begin{array}{l}\text { tissue-specific expression } \\
\text { with BIOGPS }\end{array}$ \\
\hline SLC10A2 & down-regulated & down-regulated & $\begin{array}{l}\text { solute carrier family } 10 \text { (sodium/bile acid cotransporter } \\
\text { family), member } 2\end{array}$ & n.d. ${ }^{* *}$ \\
\hline SLC13A1 & down-regulated & n.d & solute carrier family 13 (sodium/sulfate symporters), member 1 & Ileum, colon \\
\hline SLC15A1 & down-regulated & n.d & solute carrier family 15 (oligopeptide transporter), member 1 & intestine \\
\hline SLC26A3 & down-regulated & n.d & solute carrier family 26, member 3 & intestine rectum \\
\hline SLC30A10 & down-regulated & n.d & solute carrier family 30, member 10 & intestine \\
\hline SLC31A1 & down-regulated & down-regulated & solute carrier family 31 (copper transporters), member 1 & several tissues \\
\hline SLC5A1 & down-regulated & down-regulated & $\begin{array}{l}\text { solute carrier family } 5 \text { (sodium/glucose cotransporter), } \\
\text { member } 1\end{array}$ & early intestine and bladder \\
\hline SLC5A9 & down-regulated & n.d & $\begin{array}{l}\text { solute carrier family } 5 \text { (sodium/glucose cotransporter), } \\
\text { member } 9\end{array}$ & n.d.** \\
\hline SLC6A4 & down-regulated & n.d & $\begin{array}{l}\text { solute carrier family } 6 \text { (neurotransmitter transporter, } \\
\text { serotonin), member } 4\end{array}$ & n.d.** \\
\hline SLC7A9 & down-regulated & n.d & $\begin{array}{l}\text { solute carrier family } 7 \text { (cationic amino acid transporter, } \\
\text { y+ system), member } 9\end{array}$ & intestine \\
\hline SLC7A3* & n.d. & down-regulated & solute carrier family 7 (amino acid transporter) & n.d. \\
\hline $\mathrm{SLC} 2 \mathrm{~A} 1^{*}$ & n.d. & up-regulated & solute carrier family 2 (glucose tranporter) & n.d. \\
\hline
\end{tabular}


are secreted by goblet cells $[42,43]$. TFF3 is important in epithelial restitution and cell migration, though not in proliferation, while mucin and TFF3 provide mucosal protection [43]. Resistin-like beta is a cytokine with multiple functions related to mucosal integrity, immunoregulation and intestinal glucose transport [44]. CLDN15 (claudin 15) and HEPACAM2 (hepacam family member 2) were the only tight junction-associated genes affected in our study. HEPACAM2 is discussed later. Claudins are one of the four major integral membrane proteins but, pertinent to $\mathrm{PE}$, in a homozygous claudin 15 deficient mouse model of mega-intestine, there is increased proliferation of crypt cells following weaning, suggesting a role for this protein in the modulation of crypt epithelial cell turnover [45]. In this study, MUC2, TFF2, TFF3, RETNLB and CLDN15 expression levels were decreased between 3- and 9-fold at $14 \mathrm{dpc}$, phenomena that may lead to loss of mucosal integrity, disturbed cellular homeostasis and breakdown of intercellular relationships, particularly when considered in conjunction with expression changes in the SLC gene family and cubilin. As indicated above, presumably this reduced transcription is a further consequence of suppressed crypt epithelial cell maturation.

The apparent ability of $L$. intracellularis to impede the maturation of crypt enterocytes is one of the most intriguing aspects of PE, more so because it is reversible [21]. However, underlying mechanisms of pathogenesis and resolution remain undefined. McOrist et al. [21] speculated that L. intracellularis could influence genes controlling differentiation and the first support for this hypothesis was provided by $\mathrm{Oh}$ et al. [19] who reported differential expression of several genes involved in cell cycle, cellular differentiation, apoptosis and signal transduction. Up-regulation of one such gene, IGFBP3 (insulin-like growth factor binding protein 3), has been described by two separate and quite different PE studies $[19,20]$. In our study, cellular proliferation was one of the functional classes where transcriptional alterations were infrequent, with only one gene that is directly involved in cell cycle regulation, $C D K 1$, up-regulated. CDK1 drives the cell through the G2 phase of the cell cycle, which immediately precedes mitosis [17]. A link between altered CDK1 expression and PE has not been previously reported. Vannucci et al. [16] described upregulation of CDK2-associated protein 1 (CDK2AP1), a growth suppressor that down-regulates CDK2 [46]. Since CDK2 has an effect on the cell cycle that is broadly similar to CDK1, it seems counter-intuitive that a CDK2 suppressor protein is up-regulated in PE. This merits further exploration. We detected 5-fold down-regulation in the transcription of HEPACAM2, a gene encoding hepacam which is an immunoglobulin-like cell adhesion molecule purported to be a tumour suppressor [47]. HEPACAM down-regulation has been reported in several human cancers and is believed to function by down-regulating c-myc and cyclin D1 [47-50]. Thus, reduced transcription of HEPACAM2 associated with $L$. intracellularis infection could effectively "release the brakes" on c-myc and cyclin D1, leading to increased transcriptional activation and cellular proliferation. This is also an area worthy of deeper investigation.

In the context of local immunity, some of our findings are comparable with previous studies, such as the upregulation of $S L A-3$ (encodes $\mathrm{MHCI}$ ), and the downregulation of genes encoding elements of the CD3 T-cell receptor [16,22]. This apparent immunosuppression has been borne out by Jacobson et al. [20] who found limited expression of serum and tissue cytokines in natural cases of PE. Several previous studies have described macrophages in the lamina propria of infected pigs, often containing L. intracellularis organisms and sometimes peaking with maximum proliferation and burden of infection [10,22,50-53]. In our study, CD163 up-regulation at 14 dpc correlated with these previous results, although we could not convincingly confirm this immunohistochemically in tissues, possibly due to low pig numbers.

To conclude, this microarray-based study progresses the growing literature base that has more recently focused on the pathogenesis of PE, particularly at the molecular level. It provides evidence to support disruption of cell transport and mucosal integrity in pigs infected with L. intracellularis. Perhaps most interestingly, it has identified CDK1 and hepacam as potentially important molecules capable of influencing cellular proliferation in infected pigs.

\section{Additional files}

Additional file 1: Primer sequences for the microarray validation study. Primer sequences used for the QPCR validation of the microarray data. Additional file 2: List of transcripts regulated post infection with FDR $<\mathbf{0 . 1}$. List of transcripts that were significantly regulated (FDR $<0.1$ ) using Partek software, as described in Materials and methods

Additional file 3: Fold regulation of selected transcripts in ileum tissue during the experimental challenge of pigs with Lawsonia intracellularis LR189/5/83. Transcript levels were measured using qPCR as described in Materials and methods and primer sequences described in Additional file 1. Fold regulation of transcript normalised over the untreated control and GAPDH mRNA transcript level as described previously [27]. $3(n=3), 7(n=4), 14(n=4), 21(n=4), 28(n=4), 35$ $(n=2), 42(n=3) \mathrm{dpc}$.

Additional file 4: Gene ontology analysis of the data at $14 \mathrm{dpc}$ using DAVID bioinformatics resources [30]. The transcripts described in Additional file 2 were subjected to gene ontology analysis using the DAVID bioinformatics resources.

Competing interests

The authors declare they have no competing interests.

Authors' contributions

SHS, ADW, IVE contributed to the study design, acquisition, analysis of the data and manuscript preparation. IVM contributed to L. intracellularis quantification and analysis. NM contributed to the CD163

immunohistochemistry. ALA and TAA contributed to the study design, data 
analysis, supervision and manuscript preparation. All authors read and approved the final manuscript.

\section{Acknowledgements}

SHS, NM and IVE were supported by funding from the Edinburgh Campaign and the ISG. ADW, ALA and TAA were supported by BBSRC Institute Strategic Programme grants. We are indebted for the help provided by ARK-Genomics (now part of Edinburgh Genomics [54]) for the microarray hybridizations and support for the data analysis using Partek Software. We are also grateful to Professor David G.E. Smith (University of Glasgow \& Moredun Research Institute) and Dr Michelle Sait (Moredun Research Institute) for the $16 \mathrm{~S}$ rRNA pGEM ${ }^{\circledR}$-T plasmid and to Dr lan Handel of the Royal (Dick) School of Veterinary Studies for his contributions to the statistical analysis. Finally, we also thank Professor David G.E. Smith for critical reading of the manuscript.

\section{Author details}

${ }^{1}$ Royal (Dick) School of Veterinary Studies, University of Edinburgh, Easter Bush Campus, Roslin, Midlothian EH25 9RG, UK. ${ }^{2}$ The Roslin Institute, University of Edinburgh, Easter Bush Campus, Roslin, Midlothian EH25 9RG, UK.

Received: 15 October 2013 Accepted: 22 April 2014

Published: 20 May 2014

\section{References}

1. McOrist S: Defining the full costs of endemic porcine proliferative enteropathy. Vet $J$ 2005, 170:8-9.

2. Möller Jensen $\mathrm{H}$ : Health management with reduced antibiotic use - experiences of a Danish pig vet. Anim Biotechnol 2006, 17:189-194.

3. McGurrin MK, Vengust M, Arroyo LG, Baird JD: An outbreak of Lawsonia intracellularis infection in a standardbred herd in Ontario. Can Vet J 2007, 48:927-930

4. Friedman M, Bednár V, Klimes J, Smola J, Mrlík V, Literák I: Lawsonia intracellularis in rodents from pig farms with the occurrence of porcine proliferative enteropathy. Lett Appl Microbiol 2008, 47:117-121.

5. Lawson GH, Rowland AC, Roberts L, Fraser G, McCartney E: Proliferative haemorrhagic enteropathy. Res Vet Sci 1979, 27:46-51.

6. Friendship RMC, Corzo CA, Dewey CE, Blackwell T: The effect of porcine proliferative enteropathy on the introduction of gilts into recipient herds. J Swine Health Prod 2005, 13:139-142.

7. Rowland AC: Porcine intestinal adenomatosis: a possible relationship with necrotic enteritis, regional ileitis and proliferative haemorrhagic enteropathy. Vet Rec 1975, 97:178-181.

8. Rowland AC, Lawson GH: Intestinal adenomatosis in the pig: a possible relationship with a haemorrhagic enteropathy. Res Vet Sci 1975, 18:263-268

9. Lawson GH, Gebhart CJ: Proliferative enteropathy. J Comp Pathol 2000, 122:77-100

10. McOrist S, Jasni S, Mackie RA, MacIntyre N, Neef N, Lawson GH: Reproduction of porcine proliferative enteropathy with pure cultures of ileal symbiont intracellularis. Infect Immun 1993, 61:4286-4292.

11. Kroll JJ, Roof MB, McOrist S: Evaluation of protective immunity in pigs following oral administration of an avirulent live vaccine of Lawsonia intracellularis. Am J Vet Res 2004, 65:559-565.

12. Kroll JJ, Eichmeyer MA, Schaeffer ML, McOrist S, Harris DL, Roof MB: Lipopolysaccharide-based enzyme-linked immunosorbent assay for experimental use in detection of antibodies to Lawsonia intracellularis in pigs. Clin Diagn Lab Immunol 2005, 12:693-699.

13. Nogradi N, Slovis NM, Gebhart CJ, Wolfsdorf KE, McCracken JL, Scoggin CF, Kass PH, Mapes SM, Toth B, Lundquist ML, Pusterla N: Evaluation of the field efficacy of an avirulent live Lawsonia intracellularis vaccine in foals. Vet J 2012, 192:511-513.

14. Park S, Lee JB, Kim KJ, Oh YS, Kim MO, Oh YR, Hwang MA, Lee JA, Lee SW: Efficacy of a commercial live attenuated Lawsonia intracellularis vaccine in a large scale field trial in Korea. Clin Exp Vaccine Res 2013, 2:135-139.

15. Lawson GH, McOrist S, Jasni S, Mackie RA: Intracellular bacteria of porcine proliferative enteropathy cultivation and maintenance in vitro. J Clin Microbiol 1993, 31:1136-1142.

16. Vannucci FA, Foster DN, Gebhart CJ: Laser microdissection coupled with RNA-seq analysis of porcine enterocytes infected with an obligate intracellular pathogen (Lawsonia intracellularis). BMC Genomics 2013, 14:421.
17. Schafer KA: The cell cycle: a review. Vet Pathol 1998, 35:461-478,

18. Lax AJ, Thomas W: How bacteria could cause cancer: one step at a time. Trends Microbiol 2000, 10:293-299.

19. Oh YS, Lee JB, McOrist S: Microarray analysis of differential expression of cell cycle and cell differentiation genes in cells infected with Lawsonia intracellularis. Vet J 2010, 184:340-345.

20. Jacobson M, Andersson M, Lindberg R, Fossum C, Jensen-Waern M: Microarray and cytokine analyses of field cases of pigs with diarrhoea. Vet Microbiol 2011, 153:307-314.

21. McOrist S, Roberts L, Jasni S, Rowland AC, Lawson GH, Gebhart CJ, Bosworth $B$ : Developed and resolving lesions in porcine proliferative enteropathy: possible pathogenetic mechanisms. J Comp Pathol 1996, 115:35-45.

22. Maclntyre N, Smith DG, Shaw DJ, Thomson JR, Rhind SM: Immuno-pathogenesis of experimentally induced proliferative enteropathy in pigs. Vet Pathol 2003, 40:421-432.

23. Knittel JP, Jordan DM, Schwartz KJ, Janke BH, Roof MB, McOrist S, Harris DL: Evaluation of antemortem polymerase chain reaction and serologic methods for detection of Lawsonia intracellularis-exposed pigs. Am J Vet Res 1998, 59:722-726.

24. Alberdi MP, Watson E, MCAllister GEM, Harris JD, Paxton EA, Thomson JR, Smith DGE: Expression by Lawsonia intracellularis of type III secretion system components during infection. Vet Microbiol 2009, 139:298-303.

25. Lindecrona $\mathrm{RH}$, Jensen TK, Andersen PH, Møller K: Application of a 5 nuclease assay for detection of Lawsonia intracellularis in fecal samples from pigs. J Clin Microbiol 2002, 40:984-987.

26. Ait-Ali T, Wilson AD, Westcott DG, Clapperton M, Waterfall M, Mellencamp MA, Drew TW, Bishop SC, Archibald AL: Innate immune responses to replication of porcine reproductive and respiratory syndrome virus in isolated Swine alveolar macrophages. Viral Immunol 2007, 20:105-118.

27. Ait-Ali T, Wilson AD, Carré W, Westcott DG, Frossard JP, Mellencamp MA, Mouzaki D, Matika O, Waddington D, Drew TW, Bishop SC, Archibald AL: Host inhibits replication of European porcine reproductive and respiratory syndrome virus in macrophages by altering differential regulation of type-I interferon transcriptional response. Immunogenetics 2011, 63:437-448.

28. Freeman TC, Ivens A, Baillie JK, Beraldi D, Barnett MW, Dorward D, Downing A, Fairbairn L, Kapetanovic R, Raza S, Tomoiu A, Alberio R, Wu C, Su Al, Summer KM, Tuggle CK, Archibald AL, Hume DA: A gene expression atlas of the domestic pig. BMC Biol 2012, 10:90.

29. Array Express. [http://www.ebi.ac.uk/arrayexpress/]

30. DAVID Bioinformatics Resources 6.7. [http://david.abcc.ncifcrf.gov/home.jsp]

31. Ingenuity Pathway Analysis. [Www.ingenuity.com]

32. BioGPS. [www.biogps.org]

33. Schaer DJ, Schleiffenbaum B, Kurrer M, Imhof A, Bächli E, Fehr J, Moller HJ, Moestrup SK, Schaffner A: Soluble hemoglobin-haptoglobin scavenger receptor $\mathrm{CD} 163$ as a lineage-specific marker in the reactive hemophagocytic syndrome. Eur J Haematol 2005, 74:6-10.

34. Guedes RM, Gebhart CJ: Comparison of intestinal mucosa homogenate and pure culture of the homologous Lawsonia intracellularis isolate in reproducing proliferative enteropathy in swine. Vet Microbiol 2003, 93:159-166.

35. Wattanaphansak S, Gebhart C, Olin M, Deen J: Measurement of the viability of Lawsonia intracellularis. Can J Vet Res 2005, 69:265-271.

36. Alderton MR, Borland R, Coloe PJ: Experimental reproduction of porcine proliferative enteritis. J Comp Pathol 1992, 106:159-167.

37. Guedes RM, Gebhart CJ, Winkelman NL, Mackie-Nuss RA, Marsteller TA, Deen $\mathrm{J}$ : Comparison of different methods for diagnosis of porcine proliferative enteropathy. Can J Vet Res 2002, 66:99-107.

38. Huerta B, Arenas A, Carrasco L, Maldonado A, Tarradas C, Carbonero A, Perea A: Comparison of diagnostic techniques for porcine proliferative enteropathy (Lawsonia intracellularis infection). J Comp Pathol 2003, 129:179-185.

39. Choi KC, Jeung EB: Molecular mechanism of regulation of the calcium binding protein calbindin-D9k, and its physiological role(s) in mammals: a review of current research. J Cell Mol Med 2008, 12:409-420.

40. Hediger MA, Romero MF, Peng JB, Rolfs A, Takanaga H, Bruford EA: The $A B C s$ of solute carriers: physiological, pathological and therapeutic implications of human membrane transport proteins. Pflugers Arch 2004, 447:465-468.

41. Girardi AC, Di Sole F: Deciphering the mechanisms of the $\mathrm{Na}+/ \mathrm{H}+$ exchanger 3 regulation in organ dysfunction. Am J Physiol Cell Physiol 2012, 302:C1569-C1587. 
42. Thim L, May FE: Structure of mammalian trefoil factors and functional insights. Cell Mol Life Sci 2005, 62:2956-2973.

43. Kim YS, Ho SB: Intestinal goblet cells and mucins in health and disease: recent insights and progress. Curr Gastroenterol Rep 2010, 12:319-330.

44. Krimi RB, Letteron P, Chedid P, Nazaret C, Ducroc R, Marie JC: Resistin-like molecule-beta inhibits SGLT-1 activity and enhances GLUT2-dependent jejunal glucose transport. Diabetes 2009, 58:2032-2038.

45. Tamura A, Kitano Y, Hata M, Katsuno T, Moriwaki K, Sasaki H, Hayashi H, Suzuki Y, Noda T, Furuse M, Tsukita S, Tsukita S: Megaintestine in claudin-15-deficient mice. Gastroenterology 2008, 134:523-534.

46. Sun M, Jiang R, Wang G, Zhang C, Li J, Jin C, Zhang X: Cyclin-dependent kinase 2-associated protein 1 suppresses growth and tumorigenesis of lung cancer. Int J Oncol 2013, 42:1376-1382.

47. Chung Moh M, Hoon Lee L, Shen S: Cloning and characterization of hepaCAM, a novel Ig-like cell adhesion molecule suppressed in human hepatocellular carcinoma. J Hepatol 2005, 42:833-841.

48. Moh MC, Zhang T, Lee LH, Shen S: Expression of hepaCAM is downregulated in cancers and induces senescence-like growth arrest via a p53/p21-dependent pathway in human breast cancer cells. Carcinogenesis 2008, 29:2298-2305.

49. He Y, Wu X, Luo C, Wang L, Lin J: Functional significance of the hepaCAM gene in bladder cancer. BMC Cancer 2010, 10:83.

50. Wang Q, Luo C, Wu X, Du H, Song X, Fan Y: hepaCAM and p-mTOR closely correlate in bladder transitional cell carcinoma and hepaCAM expression inhibits proliferation via an AMPK/mTOR dependent pathway in human bladder cancer cells. J Urol 2013, 190:1912-1918.

51. Love DN, Love RJ: Pathology of proliferative haemorrhagic enteropathy in pigs. Vet Pathol 1979, 16:41-48.

52. Lomax $L G$, Glock RD: Naturally occurring porcine proliferative enteritis: pathologic and bacteriologic findings. Am J Vet Res 1982, 43:1608-1614.

53. Jensen $T K$, Christensen BB, Boye M: Lawsonia intracellularis infection in the large intestines of pigs. APMIS 2006, 114:255-264.

54. Edinburgh Genomics. [https://genomics.ed.ac.uk]

doi:10.1186/1297-9716-45-55

Cite this article as: Smith et al.: Down-regulation of mechanisms involved in cell transport and maintenance of mucosal integrity in pigs infected with Lawsonia intracellularis. Veterinary Research 2014 45:55.

\section{Submit your next manuscript to BioMed Central and take full advantage of:}

- Convenient online submission

- Thorough peer review

- No space constraints or color figure charges

- Immediate publication on acceptance

- Inclusion in PubMed, CAS, Scopus and Google Scholar

- Research which is freely available for redistribution

Submit your manuscript at www.biomedcentral.com/submit
(O) Biomed Central 